\title{
Utilización de métodos cuantitativos para el estudio de la dinámica de los pastos marinos: Una revisión crítica
}

\author{
About the use of quantitative methods for the study of the dynamics of seagrasses: A critical review \\ Elena Solana-Arellano \\ Centro de Investigación Científica y Educación Superior de Ensenada Baja California México. \\ P.O. box 434844, San Diego CA. 92143-4844 U.S.A \\ esolana@CICESE.mx
}

Resumen.- Se presenta una revisión sobre el tema: aplicación de métodos cuantitativos al estudio de aspectos dinámicos en poblaciones de pastos marinos. Se incluyen los modelos más importantes utilizados para describir crecimiento, edad, demografía y densidad. En algunos casos se proponen modificaciones a los modelos existentes o bien se sugieren ideas para la realización de futuras investigaciones.

Palabras clave: modelos matemáticos, dinámica, ambientes estuarinos.
Abstract.- A revision of quantitative methods applied to the study of the dynamics of seagrasses populations is presented. Included are the most important models used to describe growth, age, demography and density. In some cases, modifications to the existing models are proposed.

Suggestions for future research are also posted rendered.

Keywords: mathematical models, dynamics, estuarine environment.

\section{Introducción}

Los estudios sobre pastos marinos en ecosistemas costeros se iniciaron con los trabajos de Petersen, los cuales se reportaron en las contribuciones del mismo autor en 1914 (fide Short 1980). Sin embargo, no fue hasta la década de los cuarenta, cuando se reconoció la importancia de las grandes extensiones de estas fanerógamas en lagunas costeras de aguas templadas y tropicales ( Mann 1972).

Aún cuando los ecosistemas que se asocian con praderas de pastos marinos están ampliamente distribuidas en el globo terráqueo, el interés por su estudio se inició en la década de los sesenta cuando se les ubica dentro de los ecosistemas más ricos y productivos. De hecho Den Hartog (1971) afirma que el ciclo anual de las praderas de pastos marinos dominantes, regula en un alto grado la composición de flora, fauna $\mathrm{y}$ un gran número de elementos estructurales subordinados, particularmente epífitas y pastoreadores. Los trabajos de Wood. et al. (1969) resumen el papel de los pastos marinos en el proceso cíclico que mantienen la productividad de ecosistemas costeros y estuarios. Las conclusiones obtenidas en estos trabajos sobre estos ecosistemas indican que los pastos marinos tienen tasa de crecimiento extremadamente altas, y una productividad notoriamente elevada que normalmente oscila entre los 500 y los $1000 \mathrm{gC} / \mathrm{m}^{2} / a n ̃ o$ y ocasionalmente en algunas áreas más productivas se puede alcanzar el doble. A las hojas de algunos pastos marinos, como por ejemplo Zostera marina L., se asocian un gran número de epífitas, cuya biomasa es algunas veces igual a la de la cosecha en pie de hojas que las albergan. Pocos organismos pastorean directamente en las hojas de Z. marina, las hojas muertas y desprendidas caen y se fijan en el sedimento y esto junto con la materia detrital de las raíces mantienen el ciclo activo del sulfuro. La densidad de las hojas frena las corrientes de aguas cerca de la interfase del sedimento promoviendo la sedimentación. El sistema de raíces y rizomas se incorporan al sedimento y con ayuda de las hojas inhiben la erosión. Por lo tanto, de forma resumida, podemos decir que la producción de los pastos marinos es la base de la cadena de detritus (Zieman y Wetzel 1980).

Las praderas de pastos marinos proveen también un hábitat para numerosos organismos marinos, y juegan un papel muy importante en el reciclado de nutrientes en la costa, acelerando la fijación de nitrógeno e incrementando el flujo de nutrientes difusos hacia aguas locales (Short \& McRoy 1984). Estos ecosistemas anclan y filtran el sedimento, contribuyendo a la claridad del agua, la depositación del sedimento y la estabilidad de la línea de costa. Por todo lo anterior, el estudio de estos ecosistemas de manera descriptiva y la introducción de métodos de modelación y simulación para interpretar su dinámica resulta ser de vital importancia en aspectos de conservación.

La dinámica de una pradera de pastos marinos, debe de ser estudiada en el marco de la ecología lo que 
incluye la descripción y cuantificación de los cambios demográficos además de la forma e intensidad con que estos cambios se presentan. En este trabajo se revisa la modelación de los aspectos más importantes que describen la dinámica de dichas pradera. En esta revisión se incluye la base teórica y conceptual que permitirá la generación de modelos para la interpretación de aspectos particulares de la dinámica de pastos marinos. En este contexto, la presente contribución no solo se circunscribe a una mera descripción de resultados o al análisis crítico de los mismos sino que pretende constituirse en un receptáculo de herramientas formales para la creación de modelos en el área del conocimiento que se considera.

En este trabajo, primeramente se presenta una revisión de los modelos más representativos en la literatura sobre dinámica de pastos marinos y luego se tratan los modelos de simulación de los cuales se derivaran otros modelos importantes. Se enfatiza en el tema de modelos de crecimiento, los cuales son de gran relevancia en el estudio de en la dinámica de cualquier organismo y las relaciones alométricas. Estos modelos, a pesar de su simplicidad, fundamentaron el desarrollo de poderosos métodos cuantitativos para la realización de estudios de producción. Se discute también la relación entre biomasa e índice de área foliar la cual es ampliamente utilizada en plantas clónales terrestres, pero su estudio para ambientes marinos es escaso e inconsistente. Se estudia la relación que existe entre biomasa y densidad de tallos por unidad de área, aspecto muy importante para estudios de demografía. Se examinan también, modelos o índices para determinar la edad de plantas. La modelación del crecimiento foliar en pastos marinos en particular la del crecimiento diferencial es prácticamente nula en la literatura, por lo se analiza este tema y además se proponen algunas ideas para futuras investigaciones. Finalmente se tratan generalidades sobre los escasos modelos de demografía en pastos marinos.

\section{Estudios de modelación en pastos marinos}

En este trabajo se circunscribirá al tratamiento de modelos formales. Estos se traducen en una descripción matemática de un proceso real construido con una meta en mente. Esta meta puede ser, entre otras, analizar fenómenos biológicos para entenderlos a profundidad; estimar de manera indirecta cantidades que dependen de otras mediciones; probar hipótesis estadísticas; predecir el comportamiento de un sistema a corto o largo plazo; controlar y optimizar algunas características de estudio etc.

Como los modelos son construidos de acuerdo a un problema propuesto, y puesto que la construcción de estos es una tarea interdisciplinaria, es de vital importancia que las metas sean claras en todos los niveles del procedimiento. El modelo resultante, será juzgado o valorado en función de que estas metas se hayan cumplido. Según Odum (1972) un modelo se construye por diversas razones, entre las que destacan las de proporcionar una descripción abstracta y simplificada de algún sistema, o bien, la predicción de cambios dinámicos en el tiempo. Los modelos pueden valorarse de acuerdo con tres propiedades: a) Realismo o grado en que los enunciados matemáticos del modelo corresponden, al traducirse en palabras, a los procesos biológicos que se suponen representar. b) Precisión o capacidad del modelo de predecir cambios cuantitativos y de reproducir la dinámica observada en los datos en que se basa. c) Generalidad, se refiere a la amplitud de la aplicabilidad del modelo (i.e número de situaciones distintas en que se podría aplicar).

\section{Estudios de simulación}

La necesidad de predecir la variación de la biomasa, o bien producción u otros aspectos de la dinámica de pastos marinos y marismas han estimulado el desarrollo de modelos formales. Sin embargo, los artículos sobre modelación aplicada al estudio de estos ecosistemas son escasos. La mayoría de los trabajos reportados son modelos de simulación y con excepción del trabajo de North (1967) casi todos son una extensión del modelo de Riley (1946) el cual trata de simular la dinámica del plancton. Los estudios de simulación posteriores al trabajo de North (op cit.) se enfocan también a aspectos parciales, esto es, tratan de explicar algún factor específico del ecosistema e incluso en algunos de ello no se toman en cuenta factores o variables que contribuyen a explicar alguna característica de estudio. Sin embargo, podemos considerar a los modelos de simulación en pastos marinos como los pioneros en la modelación de estos ecosistemas.

Uno de los estudios de simulación más completos es el reportado en los trabajos de Wieger et. al (1971). Dichos autores presentaron un modelo de una pradera de marisma (Spartina) que pretende interpretar la transferencia de carbono entre catorce compartimentos o variables de estado (siete bióticas y siete abióticas). Sus resultados sobre un periodo de tiempo de cinco años muestran una gran transferencia de carbono del aire a la materia orgánica particulada y disuelta en el agua y sedimento. Al hacer un análisis de sensibilidad para esta simulación, solamente 21 de los 81 parámetros con los que se trabajó en el modelo de simulación, resultaron suficientemente sensibles para cambiar el estado estable de acumulación orgánica del sistema. Sin embargo, en este modelo no se incorporaron variables importantes como la heterogeneidad espacial o la dinámica de las 
mareas. Tampoco la heterogeneidad temporal en la distribución de la biomasa o el movimiento diario y estacional del detritus, ni tampoco el movimiento de los consumidores.

Otro modelo importante de simulación es el realizado por Reimold (1974) para Spartina . Este presenta un sistema de ecuaciones diferenciales no lineales para las variables de estado: agua, sedimento, biomasa de Spartina, detritus, microflora y detritívoros. También se simuló la remoción de Spartina por efectos de la dinámica del agua, con el propósito de evaluar la respuesta en el crecimiento y el efecto resultante en las concentraciones de fósforo para las variables de estado. Para validar la simulación se realizó un muestreo experimental y se compararon los resultados predichos con las mediciones de campo. Dicha comparación de los resultados del modelo con los datos experimentales (todos basados en las concentraciones de fósforo) hizo posible caracterizar teóricamente el flujo del fósforo. Una limitación evidente de este modelo es que se usaron variables altamente agregadas. La variable agua por ejemplo, conglomera otras variables como temperatura, composición química, turbulencia, luminosidad, contenido de sedimentos en suspensión, etc. Esto disminuye la posibilidad de caracterizar o aislar la variable específica que contribuye en mayor proporción (con relación a otras variables) a explicar el comportamiento del sistema.

Uno de los trabajos más extensos sobre simulación de la producción de praderas de Z. marina es el realizado por Short (1980). En este modelo numérico se pretende explicar cuantitativamente el mecanismo de producción de $Z$. marina, con base en un sofisticado modelo conceptual. Esta contribución, además de ser pionera, es sin duda una de las más completas ya que toma en cuenta en la simulación un gran número de factores, aunque considera que los únicos factores limitantes son la luz, la temperatura y la velocidad de la corriente. El modelo conceptual del flujo de carbón establece que dicho flujo es iniciado por asimilación fotosintética, seguido a través del sistema y posteriormente modificado por fuentes auxiliares de energía. La estructura de las unidades fotosintéticas de Z. marina incluye cinco tamaños de hoja con tasas de producción diferentes para cada talla y cada una determinada por limitaciones de luz, temperatura y velocidad de las corrientes. Los almacenamientos de raíces y rizomas son alimentados por la transferencia de carbono de las hojas a las raíces, el cual según McRoy \&. Goering (1974) es de aproximadamente 17\%. El 8\% restante determinará la tasa promedio de crecimiento de la hoja, esta tasa variará para los diferentes tamaños de hojas siendo las hojas más pequeñas las que crecen más rápido. La emergencia de hojas nuevas es un proceso estacional controlado por factores ambientales que influencian la translocación de carbono fuera de los rizomas. La biomasa de almacenamiento de la emergente aumenta hasta que alcanza el peso de la hoja más pequeña. Cuando este almacenamiento es suficientemente grande entonces una nueva hoja es anidada dentro compartimento y las hojas ya existentes se mueven una talla arriba.

Para desarrollo de modelos alternativos, resulta importante describir con cierto detalle la formula utilizada por Short (1980) en su contribución:

$$
T L=\frac{P_{0}}{P_{\max }} e^{(\alpha+\beta T) T}
$$

$T L$ es el factor limitante, $\alpha$ y $\beta$ parámetros, $P_{0}$ la producción inicial y $P_{\max }$ la tasa de producción máxima. Esta ecuación fue derivada empíricamente para expresar el efecto de la temperatura $T$ sobre la planta, sugiriendo que la producción aumenta con el incremento de temperatura hasta que se alcanza un límite máximo de $25^{\circ} \mathrm{C}$ después de este límite la fotosíntesis se inhibe y por lo tanto se reduce la asimilación de carbono por lo que se presenta una reducción de la producción (Dillon 1971) (Ley de la tolerancia con respecto a la temperatura). Para modelar los cambios estacionales en temperatura, Short (1980) utilizó una función periódica de la forma

$$
T=T_{m}+T_{D} \cos \left(\frac{2 \pi(t-\alpha)}{365}\right)
$$

Donde $T_{m}$ es la temperatura media, $T_{D}$ es la amplitud $t$ el tiempo medido en días y $\alpha$ un parámetro a ajustar.

Otro de los factores limitantes tomados en cuenta en Short (1980), es la luz. La respuesta del crecimiento a este factor limitante (LL), se modela como una tasa instantánea y se calibró con datos de producción neta de McRoy \& Goering (1974). De una manera similar al efecto de la temperatura, Z. marina (y otras plantas) aumentan su producción al aumento de la intensidad luminosa hasta alcanzar un óptimo. Cuando esté es alcanzado, la fotosíntesis se inhibe y la producción disminuye (Steel 1962). La limitación en producción debida a la intensidad luminosa $L L$ se calcula usando una doble integral sobre profundidad y fotoperiodo.

$$
L L=\int^{L} \int^{t} \frac{P^{\prime}}{P_{\text {max }}^{\prime}}
$$


Siendo $P^{\prime}$ la tasa de producción relativa, $P^{\prime}{ }_{\max }$ la tasa de producción relativa máxima. La solución a esta ecuación fue encontrada empíricamente y está dada por:

$$
L L=\frac{e^{t}}{K^{\prime} L}\left[e^{-\left(\frac{I_{0}}{I_{\text {opt }}} e^{-K^{\prime} L}\right)}-e^{\frac{I_{0}}{I_{\text {opt }}}}\right],
$$

donde $K^{\prime}$ representa el punto de extinción de la luz en el agua, $I_{0}$ la intensidad luminosa inicial y $I_{o p t}$ la intensidad luminosa óptima para la producción.

Así como en el caso de la temperatura se tomo una función coseno para modelar la estacionalidad de la intensidad luminosa, dicha función fue calibrada con datos de McRoy \& Goering (1974).

El efecto de la velocidad de las corrientes en la producción (CL) fue interpretado por Short (1980) como un modelo hidrodinámico bidimensional de la forma:

$$
C L=\frac{P^{\prime \prime}}{P_{\max }^{\prime \prime}},
$$

donde $P$ "representa la tasa de producción relativa y $P_{\max }^{\prime \prime}$ la tasa de producción relativa máxima. Al mismo tiempo, se consideró la expresión

$$
\frac{P^{\prime \prime}}{P_{\max }^{\prime \prime}}=\alpha_{1}+\beta_{1} V
$$

donde $\mathrm{V}$ es la velocidad de la corriente y $\alpha_{1}, \beta_{1}$ son parámetros a estimar.

Según Short (1980), estos tres factores limitantes, (temperatura, intensidad luminosa y velocidad de las corrientes), dan lugar a una tasa de crecimiento específica representada mediante

$$
G=P_{\max } T L \cdot L L \cdot C L
$$

Finalmente después de la pérdida de biomasa por respiración nocturna $\boldsymbol{R}$ y por erosión vegetal debida al viento (E) la fórmula para la biomasa presentada por el mismo autor es:

$$
B=B_{0} e^{(G-R)} E
$$

donde $B_{0}$ es la biomasa inicial.

Los resultados muestran algunas inconsistencias con los valores observados en dos estaciones experimentales. A pesar de sus limitaciones en cuanto a realismo y generalidad, la contribución de Short (1980) representa un avance enorme en lo que a la modelación de ecosistemas de pastos marinos se refiere. Como se infiere de las conclusiones obtenidas por Solana et al.
(1997) un modelo de simulación como el presentado por Short (op. cit.), podría mejorarse sobremanera, si se incluyera en la estructura del modelo otros factores limitantes por ejemplo nutrientes (nitratos, fosfatos, amonio, etc.) y otras fuentes de pérdida de biomasa como el pastoreo. Se sabe que las praderas de sedimentos terrígenos (en ambientes templados) son nitrógeno limitantes (Short 1987) y los substratos carbonatados en ambientes tropicales tienen limitaciones de fósforo. Mas aún, Jaques Van Monfrans et al. (1984) presenta evidencias de que el pastoreo de epífitas, invertebrados, aves y otros tiene un efecto directo en el crecimiento de $Z$. marina .

Bach (1993) presenta un estudio de simulación basado en un modelo dinámico para describir la variación estacional en crecimiento y distribución de $Z$. marina, considera factores importantes que controlan el crecimiento y la producción, como transparencia del agua, temperatura, topografía, luminosidad, profundidad y nutrientes. Este trabajo es uno de los escasos reportes sobre modelación en donde la influencia de nutrientes está incluida. La autora propone dentro de su modelo de simulación, un modelo matemático de crecimiento de Z. marina limitado por nutrientes, dicha limitación es expresada por una función que considera la concentración de nitrógeno $(\mathrm{N})$ y de fósforo $(\mathrm{P})$ como variables fundamentales. La función limitante de nitrógeno y fósforo $f(N, P)$ se representa mediante el inverso de la suma de los inversos de las funciones limitadoras asociada con cada uno de los nutrientes independientes $g(N)$ y $g(P)$ respectivamente. Este planteamiento permite considerar la acción de los dos nutrientes al mismo tiempo en contraposición con la ley de Liebig (regla del mínimo). La función $f(N, P)$ se expresa como,

$$
f(N, P)=\frac{2}{g(N)^{-1}+g(P)^{-1}}
$$

donde $g(N)$ y $g(P)$ se representan en función de las concentraciones de Nitrógeno $E[N]$ y de Fósforo $E[P]$ independientemente eso es:

$$
g(N)=\frac{E[N]-E\left[N_{\min }\right]}{E\left[N_{\text {crit. }}\right]-E\left[N_{\min }\right]},
$$

y similarmente,

$$
g(P)=\frac{E[P]-E\left[P_{\min }\right]}{E\left[P_{\text {crit. }}\right]-E\left[P_{\text {min }}\right]}
$$

Donde $E[N]$ es la concentración interna de nitrógeno, $E\left[N_{\text {min }}\right]$ es el mínimo de $E[N]$ y $E\left[N_{c r i t}\right]$ es el valor crítico de $E[N]$. La función $E[P]$ para el fósforo es equivalente. 
Debido a la falta de datos la autora no incluye en el modelo general el sub-modelo para nutrientes. Sin embargo, la sola propuesta de introducir limitación por nutrientes es un gran avance en lo que a modelación respecta. Además, la autora propone una serie de pasos y experimentos para obtener la información necesaria para conocer el valor mínimo y el valor crítico de los nutrientes y poder calibrar el modelo. Otro aspecto muy importante en este trabajo es el hecho que se tome en cuenta el movimiento del agua y la profundidad. Lo anterior es relevante debido a que pocos autores consideran que $Z$. marina toma nutrientes tanto de la columna de agua a través de las hojas como del sedimento a través de las raíces (Solana et al. 1997), además que el movimiento del agua puede desprender hojas y transportar a éstas y las hojas muertas como detritus a otras partes, lo cual debe ser considerados en un modelo de crecimiento y distribución.

Verhagem y Nienhuis (1983) realizaron un modelo de simulación para el estudio del crecimiento y la distribución estacional de $Z$. marina en el lago Grevelingen en Holanda. En este modelo incorporan influencias ambientales expresadas en términos de la intensidad luminosa, la temperatura y el movimiento del agua generado por el viento. Tomando en cuenta los altos niveles permanentes de nutrientes en las aguas intersticiales del sedimento los autores suponen que el crecimiento de $Z$. marina no es limitado por la disponibilidad de nutrientes. Para incorporar el efecto de envejecimiento de los tallos en el modelo, los autores dividen los tallos en clases de edades. De este modo, el crecimiento en un momento dado depende de la función externa instantánea y de las condiciones ambientales que prevalecen durante el periodo anterior. Los autores consideran que la división de las clases de edades entre los tallos debe basarse en el hecho de que en $Z$. marina existe una distinción entre dos formas de crecimiento de tallos. Una de crecimiento temprano que empieza en tallos vegetativos y resulta en ramas generativas y flores (tallos reproductivos) y otra de crecimiento tardío de verano (tallos vegetativos sin ramificaciones). Estas dos formas de crecimiento se subdividen en clase de edades con $L / D T$ clase de edades para tallos generativos y $M / D T$ clases de edades para tallos vegetativos. El valor $D T$ representa el incremento de tiempo de iteración para la simulación y $L$ y $M$ las edades máximas.

Los autores basan su modelo conceptual en el supuesto de que la tasa de crecimiento por unidad de biomasa ha sido descrita en términos de variables ambientales externas como luz y temperatura así como en términos de una función externa de variables de control debido a la edad. De la misma manera, suponen que la tasa relativa de descomposición o perdida de biomasa de $Z$. marina se describe como una función de la temperatura y del movimiento del agua inducida por olas, corrientes y la pendiente del sustrato. Del modelo conceptual utilizado se desprende la formula para la biomasa aérea como:

$$
B=\sum_{i=1}^{L / D T} B G_{i}+\sum_{m=1}^{M / D T} B V_{m}
$$

Donde $B G_{i}$ es la biomasa del $i$-esimo tallo generativo, $L$ representa el tiempo máximo de 70 días para tallos generativos, $B V_{m}$ es la Biomasa del m-esimo tallo vegetativo, $M$ es el tiempo máximo para tallos vegetativos, el cual se tomó como de 40 días para la simulación.

Otra parte del modelo de considerado es la que describe la tasa de cambio en la biomasa de un tallo típico de Z. marina de la siguiente manera:

$$
\begin{aligned}
& \frac{d P}{d t}=P(R G R-R D R), \\
& P(0)=P S,
\end{aligned}
$$

donde $R G R$ representa la tasa relativa de crecimiento, $R D R$ la tasa relativa de perdida y $P S$ la biomasa inicial del tallo. Estas tasas fueron representadas mediante las expresiones,

$$
\begin{gathered}
R G R=M R G R \cdot F I(I) \cdot F T_{1}(T) \cdot G S(j) \\
R D R=M R D R \cdot F T_{2}(T) \cdot F H(H) \cdot D S(j)
\end{gathered}
$$

donde los factores limitantes se definen como:

$$
\begin{gathered}
I=I_{0} e^{=k H} \\
F I(I)=\left\{\begin{array}{l}
\frac{I-I_{c}}{I_{K}-I_{c}} \text { para } 0<I<I_{k} \\
1 \quad \text { para } I>I_{k}
\end{array}\right. \\
F T_{1}(T)=\Theta_{1}^{(T-20)} \\
F T_{2}(T)=\Theta_{1}^{(T-20)} \\
F H(H)=1-\delta e^{-\lambda H} \\
G S(j)=\alpha+(1-\alpha)\left(1+(j / j a)^{2}\right)^{-1} \\
D S(j)=j / L
\end{gathered}
$$


En la tabla 1 se presenta la simbología de las variables y los parámetros considerados en las ecuaciones anteriores, las unidades de cada una de las variables así como los valores con los que fueron alimentados los modelos para la simulación los cuales provienen de (1) valores basados de mediciones directas, (2) valores basados en una combinación de mediciones directas, valores inferidos de otros valores reportados en la literatura y (3) valores considerados estimaciones razonables pero que no fueron validados.

Uno de los problemas que presenta este estudio es el hecho de la selección de las funciones específicas y parámetros dadas en la tabla antes mencionadas fueron cambiadas varias veces durante la calibración del modelo hasta obtener los resultados deseados. Además, la simulación trabajó bajo supuestos que no fueron comprobados como por ejemplo se adoptó la hipótesis de McRoy \& Goering (1974) de que las raíces se alimentan por la transferencia de carbono de la biomasa aérea y que existe una constante de proporción del carbono fijado por las hojas que es trasladado a las raíces y los rizomas. Consecuentemente, la biomasa subterránea $B S$ se calculó como, $B S=k_{1} \cdot B A$ donde $k_{1}$ es una constante de proporcionalidad y $B A$ la biomasa aérea.

También se supuso que los tallos que alcanzan edad máxima son transportados fuera de la pradera lo cual no es del todo cierto, ya que gran parte de ellos permanecen ahí para formar parte del detritus. La biomasa aérea $B A$ se expresó como la suma de todas las biomasas de las clases de edad de tallos vegetativos y tallos generativos. La biomasa por clase de edad se obtuvo multiplicando la densidad de tallos y la biomasa promedio por tallo por clase de edad.

Aún cuando el trabajo de Verhagem y Nienhuis (1983) representa uno de los modelos de simulación más completos para Z. marina, es importante hacer notar las posibles imprecisiones tanto a nivel del método de muestreo como de procedimiento. Por ejemplo, el modelo se calibró con datos de 1976 en un área de muestreo fija de $0.75 \mathrm{~m}$ de profundidad pero se utilizó un método de ensayo y error que consistió en asignar valores a los parámetros a juicio de los autores. El procedimiento también incluye un criterio de concordancia entre valores predichos y observados basado en un juicio visual y por ende subjetivo como medida de cercanía en lugar de utilizar los análisis estadísticos apropiados.

Mas aún, la gran discrepancia entre el modelo y los datos reales al final de la estación de crecimiento pudo deberse también a la poca confiabilidad de los datos ya

\section{Tabla 1}

Nomenclatura de parámetros y variables considerados en el modelo de Verhagem \& Nienhuis (1983)

Values of parameters and variables considered in Verhagen \& Nienhuis model

\begin{tabular}{|c|c|c|c|c|}
\hline \multicolumn{2}{|c|}{ Unidades } & Símbolo & \multicolumn{2}{|c|}{ Descripciór } \\
\hline$P S$ & Biomasa inicial & mg C & 2,4 & (2) \\
\hline MRGR & Tasa máxima de crec. Relativo & $d^{-1}$ & 0,08 & (1) \\
\hline$M R D R$ & Tasa máxima de perdida relativa & $\mathrm{d}^{-1}$ & 0,013 & $(2)$ \\
\hline$I$ & Intensidad de la luz & $\mathrm{Jcm}^{-2} \mathrm{~d}^{-2}$ & & \\
\hline$I_{0}$ & Valor de $I$ en la superficie & $\mathrm{Jcm}^{-2} \mathrm{~d}^{-2}$ & & (1) \\
\hline$k$ & Coeficiente de extinción & $\mathrm{m}^{-1}$ & 0,691 & (2) \\
\hline$H$ & Profundidad & $\mathrm{m}$ & & (1) \\
\hline$T$ & Temperatura del agua & ${ }^{\circ} \mathrm{C}$ & & (1) \\
\hline$\Theta_{1}$ & $\begin{array}{l}\text { Coeficiente de la temperatura para } \\
\text { el crecimiento }\end{array}$ & & 1,08 & $(2)$ \\
\hline$\Theta$ & $\begin{array}{l}\text { Coeficiente de la temperatura para } \\
\text { la descomposición }\end{array}$ & & 1,04 & $(2)$ \\
\hline$I_{c}$ & Intensidad de compensación & $\mathrm{Jcm}^{-2} \mathrm{~d}^{-2}$ & 8 & $(2)$ \\
\hline$I_{k}$ & Intensidad de saturación & $\mathrm{Jcm}^{-2} \mathrm{~d}^{-2}$ & 60 & $(2)$ \\
\hline$\delta$ & $\begin{array}{l}\text { Factor de amplificación para pérdida } \\
\text { de hojas por la acción de las olas }\end{array}$ & & 5 & (3) \\
\hline$\lambda$ & Extinción del movimiento de las olas & $\mathrm{m}^{-1}$ & 1,5 & $(2)$ \\
\hline$j$ & Edad de un tallo & d & & \\
\hline ja & Edad de un tallo adulto & d & 25 & (3) \\
\hline$\alpha$ & Coeficiente de la función de & & & \\
\hline & envejecimiento & & 0,3 & (3) \\
\hline$F I$ & $\begin{array}{l}\text { Función de la luz en la tasa de } \\
\text { crecimiento }\end{array}$ & & & \\
\hline$F T_{1}$ & $\begin{array}{l}\text { Función de la temperatura en la } \\
\text { tasa de crecimiento }\end{array}$ & & & \\
\hline$F T_{2}$ & $\begin{array}{l}\text { Función de la temperatura en la } \\
\text { tasa de perdida }\end{array}$ & & & \\
\hline$F H$ & $\begin{array}{l}\text { Influencia de profundidad en la } \\
\text { tasa de perdida de hojas por la } \\
\text { acción de las olas }\end{array}$ & & & \\
\hline GS & $\begin{array}{l}\text { Efecto del envejecimiento en la } \\
\text { tasa de crecimiento. }\end{array}$ & & & \\
\hline$D S$ & $\begin{array}{l}\text { Efecto del envejecimiento en la } \\
\text { tasa de perdida }\end{array}$ & & & \\
\hline
\end{tabular}


que la biomasa promedio obtenida de estos fue irrealisticamente alta (aproximadamente $48 \mathrm{~g}$ de peso seco libre de cenizas por metro cuadrado) o probablemente a la falta de un tamaño de muestra apropiado.

Una mayor discrepancia se encuentra entre los resultados obtenidos en la simulación para la biomasa subterránea y los datos reales. En estos resultados encontramos diferencias significativas tanto en los promedios como en las tendencias en general. Por todo los anterior creemos que es importante incorporan en el modelo supuesto corroborables así como datos confiables o en su defecto un tamaño de muestra estadísticamente adecuado para que los resultados sean científicamente aceptables.

\section{Modelos de crecimiento}

Cuando se hace referencia al crecimiento en pastos marinos, generalmente se hace alusión al aumento de biomasa por tallo o a la elongación de una hoja en un período de tiempo a partir de una marca de referencia. La mayoría de los estudios de crecimiento de pastos marino son estudios descriptivos que detallan la dinámica de crecimiento de praderas por un periodo determinado de tiempo (Sand-Jensen 1974; Jacobs 1979; Ott 1980; Aioi et al. 1981; Umebayashi 1988; Aioi \& Pollard 1993).

Uno de los primeros estudios sobre tasas de crecimiento en pastos marinos fue el realizado por Patriquin (1973) para Thalassia testudinum König. En este estudio, el autor propone un modelo lineal para la tasa promedio de crecimiento de tallos previamente marcados mediante el método de Zieman (1968). De los 50 tallos marcados solo se recuperó el 60\% para mediciones de crecimiento después de cinco días. Mas aún, en la estimación antes citada se utilizó solamente el $5 \%$ de las hojas más grandes y este 5\% fue representado solamente por 13 hojas. Esto puede ser considerado como un tamaño de muestra insuficiente. Además, aun cuando se reporta un alto coeficiente de correlación entre las variables (tasa de crecimiento y longitud máxima) no se indica el coeficiente de determinación de la regresión por mínimos cuadrados para poder validar la regresión lineal.

Brouns (1987) supone una relación exponencial entre las tasas de crecimiento de los diferentes ejes y nodos de rizomas de cinco especies de pastos marinos del Pacífico Este - Indico. Los resultados de las regresiones arrojan altos coeficientes de determinación pero no se llevó a cabo una validación estadística de tales relaciones.

Solana et al. (1997) proponen una modificación al tradicional modelo monomolecular de crecimiento. En este se incluyó un factor ambiental externo $k(t)$. El modelo propuesto está dado por la ecuación:

$$
\begin{aligned}
& \frac{d l}{d t}=k(t)\left(l_{\infty}-l(t)\right) \\
& l\left(t_{0}\right)=l_{0}
\end{aligned}
$$

donde $l(t)$ representa la longitud foliar al tiempo $t$, siendo $t_{0}$ un tiempo fijo y $l_{0}$ la longitud foliar alcanzado en dicho ese tiempo.

El factor ambiental $k(t)$ fue representado de dos maneras. Por medio de una relación funcional de la forma:

$$
k(t)=f\left(E_{1}(t), E_{2}(t), \ldots \ldots E_{n}(t)\right)
$$

donde $f\left(E_{1}(t), E_{2}(t), \ldots \ldots E_{n}(t)\right)$ es una función que satisface las condiciones del teorema de Taylor y $E_{i}(t)$ para $i=1,2, \ldots, n$ son variables ambientales. En una caracterización inicial del factor de escala $k(t)$ se supuso que este variaba suavemente. Consecuentemente, invocando del teorema de Taylor fue posible considerar una aproximación de la forma:

$k(t)=k_{0}+k_{1} E_{1}(t)+k_{2} E_{2}(t)+k_{3} E_{1}(t) E_{2}(t)$

donde $k_{1}, k_{2}$ y $k_{3}$ son constantes que se obtienen de los datos. $E_{1}(t)$ la intensidad luminosa bajo el agua $\mathrm{y}$ $E_{2}(t)$ la temperatura superficial de del agua de mar . Los resultados del ajuste de la ecuación (23) a los datos disponibles de tasa promedio de crecimiento fueron razonablemente consistentes. Sin embargo, cuando se consideraron nitratos $E_{3}(t)$ y fosfatos $E_{4}(t)$ disueltos luego de utilizar la generalización de la ecuación (25) para $k(t)$ expresada mediante la forma multilineal,

$$
k(t)=k_{0}+\sum_{i}^{4} a_{i} E_{i}(t)+\sum_{i, j}^{4} b_{i} E_{i}(t) E_{j}(t)
$$

donde $k_{0}, a_{i}, b_{i}$ con $i=1, \ldots ., 4$ son parámetros se obtuvieron excelentes resultados para el ajuste correspondiente de la ecuación (23) dando como resultado un coeficiente de determinación de 0.97. Esto permitió considerar que los nutrientes disueltos controlan la dinámica foliar en Z. marina.

Mas aún, la periodicidad explícita de $k(t)$, motivo un método alternativo para caracterizarla en términos de una serie de Fourier truncada. Esta expresa $k(t)$ mediante una función dependiente del tiempo y de este modo, es posible describir el comportamiento de la solución de la ecuación (23) para cualquier periodo de tiempo arbitrario y sin poseer mediciones de las variables ambientales. Para poder lograr lo anterior, se consideró lo propuesto por Charle-Edwards et al. (1986) 
en el sentido de que tanto la temperatura como la luminosidad siguen un modelo en términos de senos y cosenos. Además la inspección directa de las gráficas de las variables ambientales contra el tiempo nos sugiere un modelo general en términos de las sumas parciales de la serie de Fourier de la forma

$$
E_{i}(t)=E_{0 i}+\sum_{n=1}^{m} a_{n i} \operatorname{sen}\left(\frac{2 \pi}{365} n t\right)+\sum_{n=1}^{m} b_{n i} \cos \left(\frac{2 \pi}{365} n t\right)
$$

donde $E_{i}(t)$ denota cada una de las variables ambientales y $a_{n i}, b_{n i}$ y $E_{0 i}$ representan los parámetros a ser ajustados. Los resultados del ajuste de la solución de la ecuación (23) con $k(t)$ definida en términos de la ecuación (27) fueron consistentes con $r^{2}=0.95$. Este ultimo procedimiento, proporcionó una alternativa de identificación empírica de la ecuación (23).

\section{Relaciones alométricas}

Otro aspecto importante en los estudios de modelación son las relaciones alométricas que existen entre las partes de las plantas. Hamburg \& Homann (1986) utilizan un modelo alométrico sencillo para relacionar biomasa en términos del largo y el ancho de la hoja de $Z$. marina consideraron que el peso seco de una hoja (w) puede expresarse como

$$
w=a_{1} l^{a_{2}} h
$$

Duarte (1991) propone varias relaciones alométricas lineales para diferentes partes de la planta, sin embargo al igual que Hamburg \& Homann (1986) dichas relaciones alométricas tampoco se justificaron matemáticamente y/o estadísticamente. Por otra parte, los modelos se calibraron para datos mezclados de diferentes regiones del mundo sin importar diferencias especificas, tipo de muestreo, condiciones ambientales, tiempo, etc. Esto nos hace pensar que los resultados pudieran ser poco representativos.

Solana et. al. (1991) proponen un modelo alométrico más general que representa la biomasa foliar en términos de largos y anchos. Mediante el uso de la ley alométrica general (Batschelet 1975). Utilizando dicha ley alométrica y la teoría de ecuaciones diferenciales parciales lineales, se justifica formalmente la ecuación del modelo propuesto. Esta después de resolver la ecuación diferencial parcial apropiada queda dada por la siguiente relación:

$$
w=a_{1} l^{a_{2}} h^{a_{3}},
$$

siendo $w$ el peso seco (mg), l el largo (mm) y $h$ el ancho (mm) de cada hoja con $a_{1}, a_{2}$ y $a_{3}$ parámetros.
El ajuste del modelo anterior se realizó utilizando datos de un muestreo puntual. Con el propósito de investigar efectos temporales y/o espaciales, Solana et al. (1998) presentan una versión generalizada del modelo alométrico arriba descrito que incorpora dichos efectos. En este ultimo estudio, el modelo alométrico fue corroborado usando datos de un muestreo anual en la Bahía de San Quintín, México. Los resultados indican que las constantes alométricas $a_{1}, a_{2}$ y $a_{3}$ son parámetros poblacionales y por ende no son sujetos de variación espacio-temporal. La utilidad de los métodos alométrico para simplificar procedimientos de determinación de la biomasa foliar en $Z$. marina, fue demostrada fidedignamente. Mas aún, los autores demostraron también que la invariabilidad espacio-temporal de los parámetros alométricos permite la utilización de métodos no destructivos en la determinación de biomasa. Lo anterior se explica en la siguiente sección.

\section{Relaciones entre biomasa e índice de área foliar}

En general, para cualquier planta el Indice de Area foliar (L.A.I) es definido por Day et al. (1989) como el área foliar orientada horizontalmente por cada unidad de sustrato. Representa una medida de área fotosintética y resume todos los elementos que determinan la cosecha en pie i.e número de hojas por tallo, densidad de tallos, largo y ancho de las hojas, etc. (Jacobs 1979). Sin embargo, para pastos marinos y desde el punto de vista matemático, la relación que existe entre la biomasa aérea y el L.A.I no ha sido clarificada en la literatura. Para la mayoría de los autores, tal relación aparece ser lineal, pero obtienen tales conclusiones utilizando exploración visual de la gráfica de biomasa aérea contra Indice de área foliar. (Jacobs 1979, Wium-Anderson \& Borum 1984, Poumian - Tapia 1986, 1995). Otros autores, ajusta datos utilizando una regresión lineal entre las variables obteniendo coeficientes de determinación aceptables pero no proporcionan validación estadística de la bondad del ajuste. Por ejemplo, Aioi (1980) reporta un patrón unimodal para biomasa y para L.A.I para Z marina en la bahía de Odawa Japón y de ahí concluye una relación lineal entre las dos variables. En un estudio posterior, Aioi \& Pollard (1993) introduce una relación lineal entre biomasa área y L.A.I pero no presenta pruebas estadísticas de los parámetros encontrados. Mas aún, esta autora reporta una relación lineal que no pasa por el origen lo cual es conceptualmente inconsistente ya que a valores pequeños de área foliar deben corresponder valores pequeños de biomasa.

La existencia de una estrecha relación entre biomasa aérea y L.A.I es obvia. Sin embargo, dicha relación no es necesariamente lineal. De hecho en la literatura no se encuentra una demostración matemática o 
estadísticamente estricta que avale la existencia de una relación lineal entre biomasa aérea y el índice antes mencionado. Datos no publicados de Solana et al. concluyen mediante la utilización del modelo alométrico de Solana et al. $(1991 ; 1998)$ que la relación lineal antes mencionada entre las dos variables representa la parte dominante de un modelo no-lineal generalizado de la forma:

$$
w(\Omega)=c L_{a i}(\Omega)+Q\left(c, q, a_{i}, \ln \left(l_{i}\right)\right)
$$

Donde $w(\Omega)$ representa la biomasa por unidad de área $\Omega$ y donde

$$
\begin{aligned}
& Q\left(c, q, a_{i}, \ln \left(l_{i}\right)\right)=\sum_{i=1}^{N(\Omega)} E\left(c, q, a_{i}, \ln \left(l_{i}\right)\right) \\
& \mathrm{y} \operatorname{con} E\left(c, q, a_{i}, \ln \left(l_{i}\right)\right) \\
& E\left(c, q, a_{i}, \ln \left(l_{i}\right)\right)=\sum_{k=0}^{\infty} \frac{c a_{i}\left(q \ln \left(l_{i}\right)\right)^{k+1}}{(k+1) !}
\end{aligned}
$$

representa la expansión en series de potencias de la relación alométrica $w_{i}=c a_{i} e^{q \ln \left(l_{i}\right)}$ siendo $c$ y $q$ constantes positivas, $a_{i}$ el área de una hoja $w_{i}$ y $l_{i}$ el peso seco y la longitud de la hoja respectivamente.

Utilizando la ecuación no-lineal (29), Solana-Arellano et al. encontraron un mejor coeficiente de determinación, se obtuvo también una mejor predicción de la constante $c$, así como una adecuada caracterización de los residuales (normalidad, independencia, aleatoriedad etc.).

Identificar la relación entre biomasa área e Indice de Area foliar es importante ya que podríamos contar con un método predictivo no destructivo de biomasa. Esto se podría llevar a cabo debido a que con un CI-202 portable Leaf Area meter (Forestry Suppliers, Inc. Catalog 1996) o un LAI-2000 Li-Cor plant canopy analyzer, (Li-cor catalog 1997) mediciones rápidas y no destructivas de L.A.I pueden ser tomadas de manera directa sin perturbar el ecosistema y evitando trabajo de laboratorio costoso y tedioso.

\section{Relación entre densidad de tallos y biomasa}

Para estudiar la relación densidad-biomasa, es necesario estudiar la mortalidad denso-dependiente la cual se refiere al riesgo de muerte asociado el aumento en la densidad de la población de plantas. Para plantas terrestres anuales y perennes dicha relación ha sido ampliamente estudiada (Yoda et al. 1963, Harper \& White 1974, Pickard 1983) y él termino auto-aclareo se utiliza para definir la mortalidad denso-dependiente en las correspondientes poblaciones.
En su estudio sobre auto-aclareo, Yoda et. al. (1963) encuentra que la relación entre peso promedio y densidad de sobrevivientes puede escribirse como:

$$
w=c \rho^{-3 / 2} \text {. }
$$

Para plantas anuales, esta relación se conoce como regla de auto-aclareo o ley de los $-3 / 2$ y ha sido probada para plantas terrestres anuales y perennes así como para árboles de bosques.

Algunos autores (Miyanishi et al. 1979, Duarte \& Kalf 1987) conjeturan que la generalidad de la ley de los $-3 / 2$ debe ser revisada y que la relación biomasadensidad depende de las estrategias de crecimiento de la planta así como de la geometría o diferencias en las características del suelo. Miyanishin et al. (1979) propone una generalización de la ley de los $-3 / 2$ dependiendo de la estrategia de crecimiento de la planta y propone cinco estrategias hipotéticas de crecimiento en las cuales el exponente de $-3 / 2$ cambia dependiendo del modelo geométrico que se tenga.

Duarte \& Kalf (1987), hacen el primer estudio sobre la aplicabilidad de la regla de los $-3 / 2$ a pastos marinos. En su estudio consideran poblaciones sumergidas en lagos de Quebec Canadá. Los autores examinaron la influencia de las condiciones del área. En este estudio los autores resumen de manera general que la relación entre el logaritmo de la densidad y biomasa fue lineal con pendiente somera. Obtuvieron ordenadas al origen hasta 10 veces más pequeñas que las de las plantas terrestres y atribuyeron estas diferencias a los niveles de luz disponible para las plantas. Sin embargo, Olesen y Sand-Jensen (1994) en un estudio basado en 29 poblaciones de $Z$. marina encontraron que la biomasa máxima de hojas fue independiente de la densidad de tallos entre poblaciones y en concordancia con la ley de "producción final por unidad de área constante" en su estudio encuentran que la relaciona biomasa aérea y densidad siguió un patrón estacional cíclico similar al que presentan las plantas clónales terrestres con una formación continua de tallos. Además, conjeturan que la mayoría de las poblaciones de pastos aumentan en biomasa manteniendo estable la densidad de sus tallos. Concluyen que las poblaciones naturales de Z $Z$. marina estudiadas no conforman la ley de los $-3 / 2$ que se observa para plantas clonales terrestres. Sin embargo, los autores señalan que los patrones de las relaciones Biomasa-densidad son muy importantes puesto que son descriptores de cambios demográficos y de disturbios para las diferentes especies de pastos marinos. Los autores realizaron un análisis gráfico de la relación biomasa-densidad a través del tiempo. Este análisis se efectúo solo para 8 poblaciones de las cuales se contaban con datos suficientes (mediciones frecuentes 1 
o 2 meses) y encontraron que las trayectorias de biomasa densidad sugieren un patrón mas o menos cíclico. Sin embargo, señalaron que en un ciclo anual estas relaciones varían considerablemente entre estaciones y el aumento de biomasa durante el verano es debido al aumento en el tamaño de las hojas y no al aumento en densidad de tallos el cual en algunas estaciones incluso disminuye.

Se ha observado, que en plantas clonales, la densidad de tallos puede permanecer aproximadamente constante debido al recambio constante de tallos y debido también a que la mortalidad es fijada por el reclutamiento en ramas laterales y por la expansión de los rizomas para producir nuevos tallos. Por lo tanto en este tipo de plantas el auto-aclareo puede no observarse excepto para un periodo corto de verano donde se observa la biomasa máxima y la mortalidad excede la tasa de formación de tallos (Olesen \& Sand-Jensen 1994, Hutchings 1979). La estrategia de crecimiento en este tipo de plantas, las hace capaces de alterar su ciclo de crecimiento como respuesta a condiciones ambientales y/o disturbios antropogénicos por ejemplo, Olesen \& Sand-Jensen (1994) concluyen que poblaciones sujetas a una incidencia de disturbios, destinan su biomasa al incremento del tamaño de los tallos y de esa manera mantener estable la densidad de población. En contraste, la disminución de biomasa durante el periodo anterior al crecimiento asegura la disponibilidad de luz y por lo tanto la supervivencia de los tallos nuevos contribuyendo así a la recuperación de poblaciones que han sufrido disturbios.

Según Hutchchings (1979) las conexiones fisiológicas entre los tallos de un clon, deben prevenir la mortalidad denso-dependiente. En su artículo concluye que la densidad de ramets es restringida por controles ambientales y controles internos a la planta. Argumenta que el aclareo de ramets no ocurre como resultado directo de su crecimiento. Por lo tanto afirma que la ley de los $-3 / 2$ no es aplicable para poblaciones de plantas perennes con crecimiento clonal. Por otro lado, Yoda et al. (1963) definen el fenómeno de auto-aclareo como un proceso natural para plantas que tienen mortalidad denso-dependiente. Los pastos marinos y en general la plantas clónales tienen un vigoroso crecimiento vegetativo que da como resultado una extensiva expansión clonal por lo que la mortalidad de tallos en este tipo de plantas no se considera denso- dependiente. De hecho, algunos autores consideran que la mortalidad de tallos de las plantas perennes clónales es consecuencia de su estrategia de crecimiento y no de una competencia por espacio.

Hutchings (1979) aborda el problema de la estrategia de crecimiento para plantas clónales perennes. Puntualiza la relevancia del caso en el cual la gráfica de la variación temporal del logaritmo del peso promedio por planta contra el logaritmo de densidad de tallos por metro cuadrado a través del tiempo presenta una tendencia cíclica. Destaca que cuando esto ocurre, no se cumple la regla de los $-3 / 2$ y argumenta que esto sucede para todas las plantas clónales perennes que crecen bajo condiciones naturales. Asegura que este resultado se aplica siempre que exista redistribución de materia entre las diferentes partes de las plantas a las diferentes épocas del año (Hutchings \& Barkham 1976, Sonseveld 1962, Struik 1965). Aunque los trabajos de Hutchings (op. Cit) fueron realizados para plantas terrestres perennes clónales, la aplicación a los pastos marinos es inmediata puesto que estos están definidos como tales. Mas aún, se ha demostrado que los pastos marinos, (Short and McRoy 1984, Solana et al. 1998) presentan transferencia de materia entre diferentes partes de la planta como estrategia de crecimiento a través de las diferentes épocas de año. Por lo anterior podríamos concluir que la ley de auto-aclareo no se manifiesta en praderas de pastos marinos.

\section{Intervalo de Plastocrono}

En estudios de desarrollo morfológico y fisiológico de plantas completas u órganos de plantas, frecuentemente los resultados son gráficados contra la edad cronológica. Puede existir gran variabilidad entre plantas de la misma edad cronológica respecto a su desarrollo fisiológico. Además, plantas que aparentemente son morfológicamente iguales pueden ser de edad cronológica muy diferente. Mucha de esta variabilidad puede ser reducida, si en los experimentos se utilizan plantas genéticamente uniformes y además, si las condiciones experimentales son estrictamente controladas. Sin embargo en la naturaleza no podemos tener ninguna de estas dos condiciones (Harper 1977).

Con el objeto de minimizar las dificultades inherentes a los estudios de desarrollo que intentan correlacionar observaciones morfológicas y fisiológicas. Erikson y Michelini (1957) desarrollaron un índice numérico de la edad de desarrollo de plantas al cual le denominaron índice de plastocrono (abreviado PI). Este índice fue usado inicialmente para el estudio del crecimiento vegetativo de Xanathium. Sin embargo, en 1958 Michelini demostró la utilidad del índice de plastocrono utilizando, peso húmedo, peso seco, contenido de clorofila y consumo de oxigeno para hojas de Xanathium en varias etapas de su desarrollo morfológico (fide Brouns 1985).

El tiempo entre la iniciación de dos hojas consecutivas en el tallo de plantas mayores se denomina intervalo de plastocrono. Un término propuesto hace mas de un siglo en el año de 1880 por Askenasy (fide Erickson \& Michelini 1957). Estos últimos autores 
reconocieron que cuando dos plastocrones consecutivos son de igual duración, el intervalo de plastocrono puede servir como la unidad fundamental de una escala cuantitativa de desarrollo de tallos. Su índice de plastocrono esta basado en esta idea. El modelo de Erickson y Michelini (1957) es presentado en términos de la longitud de la hoja $\mathrm{n}$ y el número seriado de esa hoja como:

$$
P I=n+\frac{\log \left(l_{n}\right)-\log (r)}{\log \left(l_{n}\right)-\log \left(l_{n+1}\right)},
$$

donde $n$ es él numero seriado de la hoja y $l_{n}, l_{n+1}$ la longitud de la hoja $n$ y $n+1$ respectivamente y $r$ representa el punto de referencia. Los autores demuestran después la linealidad que existe entre la ecuación (33) y el tiempo, pudiendo así traducir plastocrones a tiempos absolutos. Finalmente los autores recomiendan que este índice formulado para italicum podría ser aplicado para estudios de crecimiento de otras plantas con las modificaciones pertinentes dependiendo de la morfología de la planta en cuestión.

Jacobs (1979) introduce para Z. marina el PI como:

$$
\text { PI = número de tallos marcados por días de exposición }
$$

La fórmula anterior, ha sido utilizada desde entonces por varios autores tanto para $Z$. marina como para otro tipo de pastos (Gallegos et al. 1992, Duarte 1991, Brouns 1985, entre otros). Cabe señalar, que en ninguno de estos escritos se da una explicación clara de cuales fueron las modificaciones pertinentes hechas a la ecuación de Erickson \& Michelini (1957) para el P.I o una derivación clara (no necesariamente matemática) de la expresión (34).

Lamoreaux et al. (1978) hace una revisión crítica del uso del P.I después de dos décadas de uso, en su estudio, hace notar tanto la gran importancia de este índice así la poca aplicación que dicho índice ha tenido en la modelación de crecimiento de plantas.

Brouns (1985) utiliza la fórmula propuesta por Jacobs (1979) y hace un estudio muy detallado para la utilización de PI en la productividad de T. hemprichii. En este estudio se estableció un límite para el error estándar de la media entre 6 al 8\% del IP medio, encontrando que el intervalo de tiempo mínimo entre el marcado y el conteo necesario para alcanzar estos limites de error fue de 10 días. Sin embargo, cabe destacar que para Z. marina al tener su hábitat en lagunas costeras debido a que en marea alta las aguas son turbias, es imposible llevar a cabo un monitoreo día a día de las hojas nuevas mediante buceo. Por lo tanto, el acopio de la información cuantitativa pertinente depende totalmente de mareas bajas. Dichas mareas, se presentan en gran parte del ciclo lunar de noche o madrugada. Aunado a esto, además del tipo fangoso de suelo en dichos hábitats tenemos el hecho de que las hojas nuevas de Z. marina son en algunas ocasiones demasiado pequeñas (hasta $7 \mathrm{~mm}$ ). Todo lo anterior, imposibilita la realización de muestreos intensivo como el propuesto por Brouns (1985).

El índice de plastocrono provee una escala de tiempo morfológica que ha demostrado ser más confiable que la edad cronológica en estudios que relacionan desarrollo morfológico y fisiológico de órganos o de una planta completa. También, proporciona una herramienta para él calculo de producción de diferentes partes de plantas como por ejemplo, producción de hojas por tallo; producción de vainas por tallo, producción de rizomas por tallo producción de raíces por tallo etc. Además, como lo señala Gallegos-Martínez (1995) el cálculo preciso del índice de plastocrono es indispensable en estudios demográficos y técnicas de reconstrucción de la edad en estos ecosistemas.

La duración media anual en días de un intervalo de plastocrono es una característica de cada especie, sin embargo, es importante estudiar la variabilidad estacional de este intervalo para cada especie ya que a esa variabilidad puede deberse las diferentes estimaciones del IP promedio reportadas en la literatura. Por ejemplo para T. hemprichii los valores promedios anuales oscilan entre 10 y 10.6 (Brouns 1985, Duarte 1991). Los valores promedio anuales del PI para $T$. testudinum se encuentran en un amplio intervalo de 14 a 24.3 (Duarte 1991, Durako 1994, Gallegos et al. 1992, Patriquin 1973). Los promedios anuales para Z. marina se presentan desde 13.1 hasta 19.3 (Duarte 1991, Jacobs 1979). Más aun valores desde 7 a 10.3 para una misma área de estudios son reportados para este pasto marino (Ibarra-Obando et al. 1997, Ibarra-Obando \& HuertaTamayo 1987, Ibarra-Obando \& Bodouresque 1994). Esta marcadas diferencias pueden deberse a la variabilidad estacional o al sistema de muestreo y conteo de las hojas nuevas en los diseños experimentales así como los días de exposición de los tallos marcados. La mayoría de los autores, obtienen sus estimaciones del PI mediante intervalos de tiempos fijos durante el periodo de estudio (generalmente 15 días).

Para Z. marina creemos que es necesario un estudio para él calculo correcto del intervalo de plastocrono basado en una modificación al muestreo de Brouns (1985) para cada estación representativa del año. De tal 
manera que se pruebe estadísticamente la probable existencia de diferencias significativas entre intervalos de plastocornos estacionales y el intervalo de plastocrono promedio anual.

\section{Crecimiento diferencial}

La relación entre crecimiento y edad, es sin duda uno de los principales temas de estudio en cualquier tipo de organismo. Para plantas en particular, la relación entre el crecimiento de hojas individuales con la edad de éstas es de gran interés en investigación. Para estudiar el crecimiento de una hoja individual necesariamente se debe conocer la tasa de crecimiento sobre períodos muy cortos de tiempo. La mayoría de los trabajos sobre el tema, plantean como crecimiento la elongación en períodos de tiempo determinado a partir de alguna marca hecha en la parte basal de la hoja, (Zieman 1974, Kentula \& McIntare 1986). Sin embargo, podríamos hacernos entre otras, las siguientes preguntas ¿en cuanto tiempo se alcanza el crecimiento máximo? ¿Es el crecimiento después de este punto no significativo? ¿Se está sub-evaluando crecimiento y por ende la producción de pastos marinos? ¿Cuánto crece una hoja y a que velocidad? ¿Cuándo deja de crecer?, etc.

Las plantas de manera típica, a diferencia de muchos animales continúan creciendo durante toda su vida. En forma continua descartan las partes viejas y añaden partes nuevas mediante crecimiento. En el proceso de crecimiento de una planta, se produce continuamente por división celular, células nuevas en un tejido especial llamado meristéma, en la periferia de estas, las células que se derivan de él crecen algunas veces hasta rebasar en muchos tantos su tamaño original y traen consigo un aumento visible en el tamaño de la planta. El crecimiento ocurre tanto en las zonas de división celular como en aquellas zonas de crecimiento de las células. A medida que estas células alcanzan su tamaño completo, dejan de crecer y se convierten en parte de los tejidos maduros de la planta. Así pues, el crecimiento de las plantas depende de zonas localizadas de división y crecimiento de las células. En contraste, en el plan de crecimiento de la mayoría de los animales, el cuerpo aumenta de tamaño junto con el conjunto de sus células en toda su extensión. Solo en las etapas más tempranas de la vida de las plantas se efectúa este tipo de crecimiento (Harper 1977). Debido a lo anterior, sería correcto suponer que cada hoja tiene un crecimiento diferencial a lo largo de ésta, esto es, se puede suponer una reducción temporal progresiva de la tasa de crecimiento tanto de la hoja en su total como también en secciones de la hoja localizadas a mayores distancias de la lígula.

Para poder modelar crecimiento y producción de pastos marinos, es necesario conocer primeramente de manera general la morfología y anatomía de la planta.
Esto con el fin de reconocer las diferentes partes que pudieran ser medidas y marcadas. Es menester también conocer la estrategia de crecimiento de la planta. La morfología general de tallos de Z. marina presentada por Tomlimson (1974) indica que estos pastos marinos están conformados de ejes vegetativos esencialmente monomórficos pero con diferenciación entre a) rizomas horizontales indeterminados y b) ejes erectos de vida corta (usualmente anuales con crecimiento determinado). La ramificación de los rizomas es monopódica con un meristéma asociado a cada hoja, la producción de ramas es difusa. Las raíces se encuentran en dos mazos en cada internodo no ramificado. Los ejes horizontales se ramifican monopódicamente. Un meristéma auxiliar en cada hoja es iniciado dentro del tercer y cuarto plastocrono. Este meristéma se separa del nodo que lo inició por medio de crecimiento intercalado y ocupa la posición de una hoja opuesta al nodo inmediatamente debajo de este. El desarrollo posterior del meristémo varía, algunos son abortados y otros son inhibidos y pueden actuar como meristémas de reserva. Otros meristémos se desarrollan como tallos con hojas los cuales pueden ser anuales con flores o como rizomas que repiten la construcción de ejes padres y de ese modo proliferan como un sistema perenne.

Como ya mencionamos anteriormente, la mayoría de los estudios de crecimiento en pastos marinos, se han llevado a cabo utilizando técnicas de marcado obtenidas modificando la técnica propuesta por Zieman (1974) (Patriquin 1973, Kentula \& McIntire 1986, Solana et al. 1997). Esta técnica consiste básicamente en marcar con una grapa metálica todas las hojas del tallo a una distancia fija del sustrato. Kentula \& McIntire 1986 modificaron la técnica de marcado de Zieman insertando una aguja hipodérmica de $0.84 \mathrm{~mm}$ a través del conjunto de hojas que conforman un tallo justamente arriba de la última vaina (lígula), pero no se encontraron diferencias significativas entre los crecimientos estimados por cada una de esas técnicas.

Las modificaciones anteriores al método original de Zieman (op. cit.) tienen en común el hecho de que el marcado del conjunto de hojas del tallo fue realizado en un solo punto (generalmente la lígula) y se ha dejado de lado el crecimiento después de esta marca. En este sentido, Patriquin (1973) estimó las tasas de crecimiento individual de cada hoja utilizando el método de Zieman (1968). Grapas orientadas verticalmente fueron colocadas a alturas de $3 \mathrm{~cm}$ en rodales de tallos cortos y a $5 \mathrm{~cm}$ en rodales de tallo grandes. Pero además, en cada una de las 10 hojas del rodal de tallos largos, fue colocada una segunda grapa a $3 \mathrm{~cm}$ de la primera y el incremento en longitud fue medido después de un período de 5 días. La longitud promedio del intervalo entre las grapas para las ocho hojas recuperadas fue de $3.2 \mathrm{~cm}$ indicando poco o cero crecimiento arriba de la primera grapa. Sin 
embargo, si consideramos que las hojas recuperadas pertenecían a tallos viejos, era de esperarse que la elongación de las hojas no fuera significativa. Además, solo se recuperó el $60 \%$ de los tallos marcados por lo que el tamaño de muestra pudiera ser no suficiente para demostrar cero crecimiento arriba de la primera grapa. Por otra parte, Zieman (1968) reporta que el 18\% de la elongación de las hojas estudiadas sé dio arriba de las grapas colocadas a $3 \mathrm{~cm}$ del sustrato lo que indica que se podría estar sub-estimando el crecimiento de cada hoja en por lo menos un $18 \%$. Un tamaño de muestra mucho más grande y en diferentes tiempos amén de una técnica diferente de marcado es requerido para modelar la tasa de crecimiento diferencial tanto de cada hoja como entre las diferentes hojas de un tallo.

Solana-Arellano (Comunicación personal) propone un método de marcado que incluya marcas a diferentes distancias en las hojas y la utilización del modelo de crecimiento general que tome en cuenta las tasas de crecimiento a las diferentes secciones de cada hoja de manera que no se subestime o sobre estime el crecimiento en un periodo de tiempo.

\section{Demografía}

El estudio de demografía es de gran importancia para conocer la dinámica poblacional de un ecosistema ya que obviamente existen diferencias demográficas importantes entre los individuos de una población. La ecología, genética, evolución desarrollo y fisiología convergen al estudio del ciclo de vida de un organismo. El ciclo de vida es una unidad fundamental en la descripción del organismo.

Las consecuencias demográficas de los ciclos de vida son fundamentales en el estudio de dinámica de poblaciones. Los pulsos vitales, (i.e tasas de nacimiento, crecimiento, desarrollo y mortalidad) de las cuales dependen las características demográficas, describen el desarrollo de los individuos a través de su ciclo de vida y la respuesta de estos ciclos al ambiente determinan la dinámica poblacional en tiempo ecológico.

Basándose en estos pulsos vitales, la demografía estudia la dinámica poblacional y la estructura de la población. El primer hecho fundamental en la dinámica poblacional es el potencial de crecimiento exponencial. Este hecho fue conocido desde el S. XVI (Cole 1958). John Grust en 1662, Linneus en 1742 y Benjamin Franklin en 1752, escribieron acerca del crecimiento exponencial, aunque el crédito se le atribuye a Mathus quien publicó "Principios de poblaciones" en 1798 (fide. Caswell 1989). El segundo hecho fundamental en dinámica poblacional, es que las poblaciones no tienen crecimiento exponencial irrestricto, esto es, una población no crece indefinidamente. Los primeros modelos de crecimiento restringido se le atribuyen a Pierre-Francois Verhulst quien derivó la ecuación logística en 1838 (fide. Hutchinson 1978), esta ecuación fue retomada y "popularizada" por Pearl \& Reed en 1920. Extensiones a modelos de competencia intraespecifica e interacciones depredador-presa surgieron rápidamente. La demografía moderna descansa en los trabajos de A.J. Lotka el cual desarrolla la teoría que relaciona la estructura y dinámica de la población clasificada por clases de edades y viviendo en ambientes constantes. Con esta teoría se deja a un lado el problema de la ecuación logística de ignorar la estructura de la población, esto es, considerar a todos los individuos como idénticos.

La demografía de plantas terrestres ha sido estudiada intensamente $\mathrm{y}$ ha permitido el reconocimiento de patrones generales de nacimiento, densidad y mortalidad de tallos (Lovett Dost 1981, Schmid \& Harper 1985) y a la formulación de teorías generales sobre la relación de biomasa-densidad en monocultivos de edades iguales. Sin embargo, aún cuando las praderas de pastos marinos son muy similares a las praderas de pastos terrestres, lo cual proporciona una gran

oportunidad de comparación entre ecosistemas terrestres y marinos, los estudios de demografía de pastos marinos son prácticamente nulos.

Uno de los primeros estudios con tendencia demográfica para pastos marinos, fue el trabajo de Petersen (1914) en el cual utilizó una secuencia de longitudes internodales de Z. marina para estimar la productividad en aguas Danesas. El trabajo de Patriquin (1973) para T. testudinum en el cual estudia tasas de crecimiento y máxima longitud así como tasas de producción aérea y subterránea y edad de la planta, sentó las ideas básicas para hacer estudios demográficos y demostró como la determinación de la edad es importante para el estudio de dinámica de pastos marinos. Demografía se define como el Estudio de cambios dinámicos, en tallos, densidades y distribuciones de poblaciones para entender los procesos como nacimientos, muertes inmigración y emigración los cuales determinan él numero de individuos en una población (Purves et al. 1992). Olesen \& Sand-Jensen en 1994 presentan el primer estudio demográfico en $Z$. marina en el cual utilizan método de cohortes para describir la relación de crecimiento y edad del tallo (en termino de plastocrones), producción estacional, pérdida de biomasa aérea, reclutamiento y mortalidad entre otras cosas. Este trabajo representa sin lugar a dudas un increíble avance en cuanto al estudio demográfico de estos ecosistemas se refiere. Es evidente que los estudios demográficos en pastos marinos son de vital importancia. Sin embargo, los trabajos publicados en este tema son escasos y la mayoría están hechos para $T$. 
testudinum y C. nodosa. (Duarte et al. 1994, GallegosMartinez et al. 1993,1994 ). Para Z. marina el trabajo de Petersen (1914) y el trabajo de Olesen \& SandJensen en 1994 son prácticamente los únicos sobre demografía de esta planta. En este último se utiliza el método de las cohortes para describir la relación entre crecimiento y edad del tallo (en término de plastocrones), producción estacional, tasas de pérdida de biomasa aérea, reclutamiento y mortalidad entre otras cosas. En particular, para las praderas de Z. marina en México el único estudio de carácter demográfico es el realizado por Poumian-Tapia \& Ibarra-Obando (1999). Sin embargo, el trabajo presenta una descripción amplia de las características de una pradera pero los pulsos vitales que describimos anteriormente como tasa de reclutamiento, tasa de mortalidad, edad de un rizoma, edad de un tallo, edad de una hoja etc., no son calculados.

\section{Conclusiones}

Aun cuando los estudios sobre pastos marinos empezaron desde 1891 (Short 1980), la modelación matemática de estos, da sus primeros frutos hasta fines de los sesenta (Mann 1972). Muchos de los modelos planteados desde entonces, carecen de rigor estadístico y matemático y otros carecen de datos para su calibración (ej: Bach 1993, Patriquin 1973, Short 1980 Brouns 1987). Los supuestos bajo los cuales se plantean las ecuaciones de algunos modelos no han sido revisados minuciosamente o, en su defecto, no han sido probados, por lo que la validez del modelo (ej: Verhagem y Nienhuis 1983, Short 1980, Reimold 1974) es en ocasiones dudosa, ya que esta basada en suposiciones irreales. Otros modelos como la ley de auto-aclareo, y el intervalo de plastocrono han sido ampliamente probados para plantas terrestres y su aplicación a pastos marinos o plantas acuáticas no han sido suficientemente examinadas (Hutchings 1979, Erickson \& Michelini 1957, Yoda et al. 1963, entre otros). Por otro lado, algunas características importantes sobre la dinámica de estos ecosistemas como la edad de un tallo o la edad de una pradera o el papel de los nutrientes en el crecimiento de algunos pastos, no han sido modeladas o han sido tocados superficialmente. En este trabajo, se ha presentado una revisión exhaustiva de los principales modelos que se han desarrollado para el estudio de pastos marinos. La necesidad de predecir la dinámica de estos ecosistemas con métodos confiables no destructivos que ayuden a su preservación y conservación hace de la modelación matemática una área imprescindible abierta a la investigación. Las 34 ecuaciones presentadas, proporcionan el marco teórico que permitirá el desarrollo de nuevos modelos para estudios específicos.

\section{Agradecimientos}

La autora agradece profundamente a la Dra. Margarita Gallegos Martínez y al Dr. Hector Echavarria Heras por la revisión de este trabajo. También a Olga Flores Uzeta y Edwina Nieto García por su valiosa ayuda en la búsqueda bibliográfica. Este trabajo fue auspiciado parcialmente por CONACYT bajo el proyecto número 26665-B.

\section{Literatura citada}

Aioi Keiko. 1980. Seasonal Change in the standing crop of eelgrass (Zostera marina L.) In Odawa Bay, Central Japan. Aquatic Botany, 8:343-354.

Aioi K, H. Mukai, I. Koike, M. Ohtsu \& A. Hattori. 1981. Growth and organic production of eelgrass (Zosterra marina L.) in temperate waters of the pacific coast of Japan. II Growth analysis in winter. Aquatic Botany, 10:175-182.

Aioi K \& PC Pollard 1993. Biomass, leaf growth and loss rate of the seagrass Syringodium isoetifolium on Dravuni Island, Fiji. Aquatic Botany,46:283-292.

Bach Hanne K. 1993. A dynamic model describing the seasonal variation in growth and distribution of eelgrass (Zostera marina L.). Ecological Modelling, 65:31-50

Batschelet, E. 1975. Introduction to mathemathics for life scientist. Springer Verlag, pp. 362-364.

Brouns JWM. 1985. The plastochron interval for the study of the productivity of seagrasses, Possibilities and limitations. Aquatic Botany. 21:71-88.

Brouns JWM. 1987. Growth patterns in some Indo-WestPacific seagrasses. Aquatic Botany,28:39-61.

Charles-Edwards DA, D Doley, \& GM Rimmington. 1986. Modelling Growth plant an developement. Academic. Press Inc. 229 pp.

Caswell H. 1989. Matrix Population Models. Sinauer Association, Inc. Publishers. Sunderland Massachusetts.

Cole LC. 1958. En: Caswell Hall. 1989. Matrix Population Models. Sinauer Association, Inc. Publishers. Sunderland Massachusetts.

Den Hartog C. 1971. The dynamic aspect of the ecology of sea-grass communities. Conference paper. Thalassia Jugoslavica 7(1): 101-112.

Day J, CAS. Hall, WM. Kempt \& A. Yañez-Arancibia. 1989. Estuarine of the Woeld. John Wiley \& Sons.226253.

Dillon CR. 1971. A comparative study of the primary production of stuarine phytoplankton and macrobenthic plants. Ph. D. Thesis, University of North Carolina, Chapel Hill N.C. 112pp.

Duarte CM. 1991. Allometric scaling of seagrass form and productivity. Mar. Ecol. Prog. Ser. Vol. 77: 289-300.

Duarte C, N Marba, N Agawin, J Cebrián, S Enríquez, MD. Fortes, M Gallegos, M Merino, B Olesen, K SandJensen, J Uri,\& J Vermaat. 1994. Reconstruction of 
Seagrass dynamics: age determination and associated tools for the seagrasses ecologist. Marine Ecology Progress Series. 107:195-209.

Duarte C \& K Sand-Jensen. 1990. Seagrass colonization: Biomass development and shoot demography in Cymodocea nodosa, patches Marine Ecology Progress Series. 67: 97-103.

Duarte CM \& J Kaff. 1987. Weigth-density relatioships in submerged macrophytes. Oecology 72:612-617 .

Durako MJ. 1994. Seagrass die-off in Florida Bay (USA): changes in shoot demography characteristics and population dynamics in Tallasia testudinum. Marine Ecology Progress Series. 110:59-66.

Erickson RO \& FJ Michelini. 1957. The plastochron index . Amer. Jor. Bot. Vol 44. pp 572-579.

Gallegos M. E, Martin Merino, Nuria Marba and Carlos Duarte. 1992. Flowering of Thalassia testudinum Banks ex Konig in the Mexican Caribbean: age-dependence and interannual variability. Aquatic Botany 43: 249-255.

Gallegos Martínez M, M Merino, N Marbá \& CM Duarte. 1993. Biomass and Dynamics of Thalassia testudinum in the Mexican Caribbean: elucidating rhizome growth. Marine Ecology Progress Series. 95:185-192.

Gallegos Martínez M, M Merino, A Rodriguez, N Marbá \& C Duarte. 1994. Growth patterns and demography of pioneer Caribbean seagrasses Halodule wrightii and Syringodiun filiforme K . Marine Ecology Progress Series 109:99-104

Gallegos Martínez ME. 1995. Dinámica de poblaciones y crecimiento de los pastos marinos caribeños Thallasia testudinum, Banks ex König, Sysringodium filliforme Kutz. Y Halodule wrightii Ascherson. Tesis de Doctorado. UNAM.

Hamburg, SP. \& PS Homann. 1986. Utilization of growth parameters of eelgrass, Zostera marina, for productivity estimation under laboratory and in situ conditions. Marine Biology. 93,pp. 299-303.

Harper JL \& J White. 1974. The demography of plants. Annual Review of Ecology and Systemathics. 5:419-63.

Harper JL.1977. Population Biology Of Plants. Academic Press. 892pp.

Hutchings MJ \& JP Barkham. 1976. An investigation of shoot interaction in Mercirialis perinnis L. a rhizomatous perennial herb. Journal of Ecology 64:723-743.

Hutchings M J. 1979. Weight-density relationship in ramet populations of clonal perennial herbs. With special reference to the $-3 / 2$ power law. Journal of Ecology. 67:21-33.

Hutchinson. GE. 1978. En: Caswell H. 1989. Matrix Population Models. Sinauer Association, Inc. Publishers. Sunderland Massachusetts.

Ibarra-Obando SE \& A Escoffet. 1987. Industria Development on the Ecology of a Pacific Mexican Estuary. Environmental Conservation. 14:135-141.

Ibarra-Obando SE.\& R Huerta-Tamayo. 1987. Blade production of Zostera marina L. during the summer- autumn period on the Pacific coast of Mexico. Aquatic Botany. 28:301-315.

Ibarra-Obando SE \& CF Boudouresque. 1994. An improvement of Zieman leaf marking technique Zostera marina growth and production assesment. Aquatic Botany 47:293-302.

Ibarra-Obando SE ,CF Boudouresque \& Maurice Roux. 1997. Leaf dynamics and production of a Zostera marina bed near its southern distributional limit. Aquatic Botany 58:99-112.

Jacobs. RPWM. 1979. Distribution and aspects of the production and biomass of eelgrass, Zostera marina. L. at Roscoff, France. Aquatic. Botany. 7:151-172.

Jacques VM, RL. Wetzel \& RJ. Orth. 1984. Epiphytes grazers relationship in seagrass meadows. Consequences for seagrass growth and reproducction. Estuarine Vol. 7 4A pp. 209-309.

Kentula. ME. \& CD. McIntire. 1986. The autoecology and production dynamics of eelgrass (Zostera marina. L.) In Netarts Bay, Oregon. Esturies. 9:188-199.

Lamoreaux, RJ, WR Chaney \& KB Brown. 1978. The plastochron index: A review after two decades of use. American Journal of Botany. 65(5): 586-593.

Lovett DL. 1981. Population dynamics and local specialization in a clonal perenial (Ranunculus repens). I. The dynamics of ramets in contrasting habitats. Journal of Ecology. 62: pp. 97- 105.

Mann KH. 1972. Macrophyte production and detritus food chain in coastal waters. Memories of . Institute Italian of Idrobiology. 29(suppl) 353-383.

McRoy CP \& JJ Goering. 1974. Nitrogen kinetics in aquatic plants artic Alaska. Aquatic Botany 1:3-10.

Miyanishi, K, AR Hot \& PB Cavers. 1979. A generalized law of self-thinning in plant populations (self-thinning in plant populations). Journal of Theoretical. Biology. 78:439-442.

North WJ. 1967. Intergration of environmental conditions by marine organisms. In: Pollution and marine ecology. Olsen T. A. (ed). Wiley and Sons. New York.

Odum EP. 1972. Ecología. Nueva Editorial Interamericana S.A. de C.V. tercera edición. 639 pp.

Olesen B. \& K. Sand-Jensen. 1994. Biomass-Density patterns in the temperate seagrass Zostera marina. Marine. Ecology. Progres. Series. 109:283-1994.

Ott J. 1980. Growth and production in Posidonia Oceanica (L.) Delile. Marine Ecology 1:47-64.

Patriquin R. 1973. Estimation of growth rate, production and age of the marine angiosperm Thalassia testudinum könig. Caribean. Journalof Sciences. 13(1-2):111-123.

Petersen CJG. 1891. Fiskenes biologiske forhold i Holbaek fjord. Rep. Danish. Biol. Sta. I:1-63.

Petersen CJG. 1914. Om Baendeltangens (Zostera narina) Aars-Produktion j de danske farvande. Mideskr. Steenstr, Fds. Kbh. 9:1-20 
Pearl R \& LJ. Reed 1920. En: Caswell Hall. 1989. Matrix Population Models. Sinauer Association, Inc. Publishers. Sunderland Massachusetts.

Pickard WF. 1983. Three interpretations of the sel-thinning rule. Annals of Botany. 51:749-757

Poumian-Tapia M. 1986. Biomasa aérea de Zostera marina L. y sus epífitas microscópicas en Bahía Falsa, San Quintín, B.C. de junio a diciembre de 1982. Tesis profecional. Escuela Superior de Ciencias Marinas. Universidad Autónoma de Baja California, Mex. pp. 87

Poumian-Tapia M. 1995. Sobre la cuantificación de la biomasa de Zostera marina L. en Bahía San Quintín, B.C. Durante un ciclo anual. Tésis de Maestría CICESE. México pp. 152.

Poumina-Tapia M. \& SE Ibarra-Obando.1999. Demography and biomass of the seasgrass Zosttera marina in a Mexican Coastal lagoon. Estuaries 22:837-847.

Purves WK, HG. Orians \& HC Heller. 1992. Life. The Science of Biology. Sinauer Associates, Inc. W.H. Freeman and Company. USA

Reimold HJ. 1974. Mathematical model in Spartina In: Ecology of Hallophytes. (Eds. R.J. Reimold and W.H Qeen. Academic Press Inc. P 393-406.

Riley GA.1946. Factors contrilling Phytoplankton pupulations on Georgia Bakn. Journal of Marine Reaserch. 6(1):5472.

Sand-Jensen, K. 1975. Biomass, net production and growth dynamics in an eelgrass (Zostera marina. L.) population in Vellerup Vig. Denmark. Ophelia 14: 185-201.

Schmid. B.\& JL Harper. (1985). Clonal growth in grasslands perennials.I. Density and pattern-dependent competition between plants with deferent growth forms Journal of Ecology. 73: pp 793-808.

Short FT. 1980. A simulation model of seagrass production systems: In Phillips R.C. McRoy C. P. (ed). Handbook of seagrass biology on ecosystems perspective. Garland New York, pp 277-295.

Short FT. 1987. Effects of sediments nutrients of seagrass: Literature review and mesocosm experiment. Aquatic Botany, 27 pp 41-57.

Short FT. \& CP. McRoy. 1984. Nitrogen uptake by leaves and roots of the segrass Zostera marina L. Botanica marina. 27:547-555.

Solana-Arellano ME, SE Ibarra-Obando \& H EchavarríaHeras. 1991. Calibración de un modelo alométrico para evaluar la producción foliar de Zostera marina L. Hidrobiológica Vol. 1(2), pp 41-44.

Solana-Arellano ME, H. Echavarria-Heras \& SE IbarraObando. 1997. Leaf-size dynamics for Zostera marina. L in San Quintin Bay, Mexico: a theoretical study. Estuarine, Coastal and Shelf Science. 44:351-359.
Solana-Arellano ME, DJ Borbon-Gonzalez \& $\mathrm{H}$ Echavarria-Heras. 1998. A general allometric model for blade production of Zostera marina L. Bulletin of the Southern California Academy of Sciences. 97:39-49

Sonselved, A. 1962. Diastribution and re-distribution of dry matter in perennial fodder crops. Netherlands Journal of Agricultural Sciences. 10:427-444

Steel, JH. 1962. Environmental control of photosynthesis in the sea. Oceanography, 7:137:150.

Struik GJ. 1965. Growth patterns of some native annual and perennial crops in Southern Wisconsin. Ecology, 58:467485.

Tomlimson PB. 1974. Vegetative morfology and meristem dependence-The foundation of productivity in seagrasses. Aquaculture

Umebayashi O. 1988. Individual growth of vegetative shoots of Eelgrass Zostera marina. Bulletin of Tokai Regional Fisheries Research Laboratory. No. 125

Verhagem, JHG \& P. Nienhuis. 1983. A simulation model of production, seasonal changes in biomass and distribution of eelgrass (Zostera marina) in lake Grevelingen. Marine Ecology Progress Series, 10:187195.

Wieger RG, RR. Christensen, J L Gallagher, JR Hall, RDH Jones \& RL Wetzel. 1971. A preliminar ecosystem model of a coastal Georgia Spartina salt marsh.: In L. E. Crininn (ed) Estuarine Reserch. Academy Press. New York. pp 583-601.

Wium-Anderson, S \& J Borum. 1984. Biomass and production of eelgrass (Zostera marina L. ) in the Oresund, Denmark. Ophelia Suppl. 1,p. 49-55.

Wood E J, WE Odum W \& JC Zieman. 1969. Influence of seagrass on productivity of coastal lagoons. In Coastal lagoons, A symposium, UNAM-UNESCO. Universidad Nacional Autónoma de Mexico. Mexico D.F. pp 459-502.

Yoda K, T Kira, H Ogawa \& K Huzumi. 1963. Self thinning in overcrowded pure stands under cultivated and natural conditions. Journal of . Biology Osawa City University Series. D 14:107-129.

Zieman JC. 1968. Study of the growth and decomposition of the sea-grass Thalassia testudinum. MSc thesis, University of Miami 50 p.

Zieman JC. 1974. Methods for the study of the growth and production of turtle grass, Thalassia testudinum Konig. Aquaculture 4: 139-143.

Zieman JC. and RG Wetzel (1980). Productivity in Seagrasses: Methods and rates. In Phillips R.C. McRoy C. P. (ed). Handbook of seagrass biology on ecosystems perspective. Garland New York, pp 87-116. 